\title{
Planar column collapse of elongated grains
}

\author{
Andrea Jara $^{1}$ and Miguel Cabrera ${ }^{1, *}$ \\ ${ }^{1}$ Department of Civil and Environmental Engineering, Universidad de los Andes, Bogotá, Colombia
}

\begin{abstract}
The granular column collapse is a benchmark configuration for the study of granular flows in dry, saturated, and submerged conditions. The collapse sequence and resultant mobility is acknowledged to be controlled by the column aspect ratio, while grain properties define the relative transition of each stage. Grain shape effects are found to modify the global shear resistance of granular media, with a strong and coupled interaction when interacting with a fluid. In this work, we present the first steps towards the study of grain shape effects in a column collapse when interacting with an ambient fluid. For this purpose, we use a planar configuration and explore the collapse of a column consisting of rod-like grains and study the initial and after collapse grain orientations. On it, the mobilized grains deposit in a preferential horizontal orientation, but further experiments are required to confirm if a nematic configuration can be achieved.
\end{abstract}

\section{Introduction}

The column setup consists of a granular material, over a horizontal surface, of initial height $H_{0}$ and initial width $L_{0}$, resulting in an initial aspect ratio $\mathcal{A}=H_{0} / L_{0}$. The sudden removal of the column confinement leads the granular material to an unstable configuration and results in a gravity driven free-fall and granular side spread [1]. This configuration was common in fluid mechanics, also known as the dam-break configuration [2-4], focusing on the fluid front propagation after release and exploring aspect ratios commonly below unity. In 2004 this configuration was introduced to the granular media community by the simultaneous publications from Lajeunesse $e t$ al. and Lube $e t a l$. $[5,6]$, exploring the collapse of dry axisymmetric granular columns as a function of $\mathcal{A}$. The observations on the column kinematics and deposit configuration sparked a rapid acceptance of the granular column as a benchmark case for the study of granular flows, drawing attention to its simple reproduction and convenient configuration for the validation of discrete and continuum numerical methods [7-10]. Despite the extensive studies on this configuration, grain size and grain shape effects remain a challenging point on proving the universality of the observations performed in experiments [11].

Grain shapes, different to nearly spherical beads, are common in pharmaceutical and food processing industries, in geophysical scenarios, among many others. High grain angularity is found to increase the global shear resistance of granular media [12]. In particular, columns with elongated or rod-like grains are found to behave similarly to columns with spherical grains, when the rod aspect ratio $\left(\mathcal{R}=l_{q} / d_{q}\right)$ is small, and transition to a non-collapsing configuration at relative high rod aspect ratios $[13,14]$. In the case of small $\mathcal{R}$, the column aspect ratio $\mathcal{A}$ would

*e-mail: ma.cabrera140@uniandes.edu.co

A video is available at https://doi.org/10.48448/0ppn-ca89 lead to the release of a full-width wedge in sufficiently tall columns and the release of an edge wedge in the case of short columns. While if $\mathcal{R}$ is large enough, the interlocking between grains would lead to the column stability, even for the case of tall columns [13]. These previous studies have proven to be useful in the understanding of the varying interlocking contact networks as a function of $\mathcal{R}$, but insights of the column internal kinematics, when interacting with an ambient fluid, remain to be explored.

In this work, we present the first steps towards the study of grain shape effects when interacting with an ambient fluid. For this purpose, we use the planar granular column setup described in [15] and explore the collapse of a column consisting of rod-like grains. The main goal is to compare the effect of the ambient fluid in the grains orientation prior and after collapse, and asses the feasibility of the experimental setup for studying grain shape effects. The paper is organized as follows. The experimental setup, granular material, and measurement methods are presented in Sec. 2. The influence of the ambient fluid in the collapse kinematics and grain orientation is presented in Sec. 3. Finally, the main conclusions and possibilities for future works are summarized in Sec. 4 .

\section{Methods}

The granular column experiments are performed in the planar setup developed by Pinzon \& Cabrera (see Fig. 1), and employed in the study of submerged column collapses $[15,16]$ and landslide generated waves [17]. In this setup, the model PMMA side windows are separated by a thin gap, confining the granular material to a nearly two-dimensional configuration. The model inner dimensions are $390 \mathrm{~mm}$ long by $390 \mathrm{~mm}$ high. The experimental setup is filled with fluid through a side-hole located at the lower right corner. The fluid is a mixture of tap water 


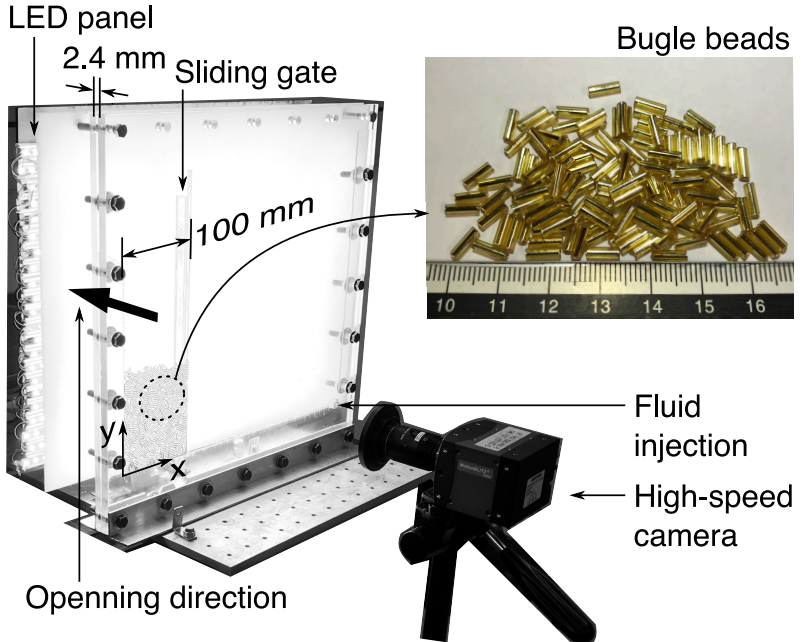

Figure 1. Planar column setup of a dry column collapse and a close-up view of the elongated grains.

and dishwashing liquid, in a concentration of 1:4\% in volume, decreasing the fluid surface tension and facilitating the column saturation.

A $4000 \mathrm{~lm}$ LED panel backlights the experimental setup and a high-speed camera records the experiments at a frame-rate of $500 \mathrm{~Hz}$ and with a resolution of $1280 \mathrm{px}$ by 1024 px. The experimental space is calibrated using marks on the model perimeter, yielding a calibration factor of $\sim 0.21 \mathrm{~mm} / \mathrm{px}$ and allowing the use of a depthcorrection algorithm for potential image perspective errors. The opening mechanism consists of a thin horizontal sliding gate that rapidly passes through a narrow $0.2 \mathrm{~mm}$ slot in the rear PMMA window and is activated by a linear pneumatic actuator, operating under a pressure of 4 bar. The gate opens horizontally in a direction normal to the $x y$ plane (see Fig. 1), releasing all grains simultaneously. For more details please see Refs. [15, 17, 18].

The granular material consists of tube-shaped glass beads, also known as bugle beads, of mean grain diameter $d_{\mathrm{g}}=2.15 \pm 0.04 \mathrm{~mm}$, grain length $l_{\mathrm{g}}=6.92 \pm$ $0.18 \mathrm{~mm}$, inner diameter of $0.6 \mathrm{~mm}$, and grain density $\rho_{\mathrm{g}}=1839 \mathrm{~kg} / \mathrm{m}^{3}$ (see Fig. 1). The beads have a glass translucent exterior and inner gold coating, providing a clear orientational pattern for image analysis. The grain aspect ratio $(\mathcal{R}=3.21)$ is close to the intermediate shape effects identified by Trepanier and Franklin, where the column collapse could not be predicted a priori [13].

The column is built towards one of the model sides, within an initial width $L_{0}=100 \mathrm{~mm}$ and releasing the beads from a height of $410 \mathrm{~mm}$, until reaching an initial height of $H_{0}=110 \mathrm{~mm}$. The formation of grain clusters with side-to-side contacts is recurrent and intrinsic to the model preparation. Several attempts in breaking such initial configuration were futile, leaving the initial packing configuration as aleatory as possible. A single column aspect ratio $\mathcal{A}=1.1$ is employed in this work, focusing on the collapse sequence and its interaction with the ambient fluid. In consequence, two series of experiments are explored, performing five repetitions for the dry columns and three repetitions for the submerged columns, while preserving the same number of beads within the model for each series.

For the submerged experiments, a $2.5 \mathrm{~mm}$ O-ring seals the perimeter of the inner gap and the fluid level is raised $70 \mathrm{~mm}$ above the column height (see Fig. 2). The column stability is found to strongly depend on the saturation process during preparation, resulting in unwanted suction between the grains and the inner walls when poor or quick saturation was performed. For these reasons, special care is taken in removing trapped air bubbles during saturation by means of slow fluid injection and internal grain shaking after submergence.

\section{Results and discussion}

As pointed by Trepanier and Franklin, the column collapse of rods is similar to that of spheres or sand, but with an irregular release of grains as a function of the rod aspect ratio $\mathcal{R}$ [13]. Here, we identify that for the specific conditions of the planar setup and rod aspect ratios, the column collapse occurs as the release of a top wedge, falling and spreading laterally, while maintaining an inner and extensive basal stagnant core.

Dry columns collapse with the release of a triangular top wedge, followed by the collective free-fall of grains that rebound against the model base and transition into a collective side spreading (see Fig. 2). The deposits do not present an unique slope, in consequence two regions are identified, a top steep slope of stagnant grains and a basal moderate slope of mobilized grains (see Fig. 2(e)). Interestingly, the amount of mobilized grains control the resultant collapse kinematics. The wedge consists of $\sim 80$ grains and is strongly controlled by the stabilization effect of the side-to-side clusters near the column free-face.

Submerged columns collapse with the mobilization of fewer grains $(\sim 40)$, falling from the released top wedge and accumulating at the base with minimal side-spreading and no rebounds (see Fig. 2). It is noticeable the extent of drag acting on the released grains, both controlling the falling velocity and grain orientation, reflected in the limited deposit mobility. Isolated grains present a side-toside oscilation, also called fluttering [19], marking a transition towards a horizontal orientation when reaching the deposit. The deposits present an interrupted slope, similar to the dry case, but with a stronger stabilization support from the side-to-side clusters that result in steeper deposits (see Fig. 2(j)).

The grain orientation is extracted by means of digital image analysis, using the scikit-image library [20]. The analysis focuses on the initial and final snapshots of each experiment, aiming at collecting the grain orientation $(\vartheta \in[0, \pi])$ in the image two-dimensional plane. The image analysis is performed in two steps. First, the image is binarized, with the Sauvola threshold [21], enhancing the contrast between the grains and the background lighting (see Fig. 2(b)). And second, the regionprops module isolates the grains and measures the grain orientation, length, width, among others (see Fig. 2(c)). The method is inefficient for cases of non-uniform backlighting or when 


\section{Dry collapse}

(a) $t=0 \mathrm{~s}$

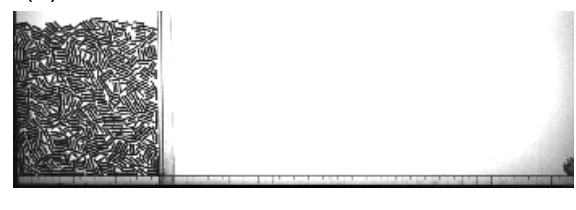

(b) $t=0.154 \mathrm{~s}$

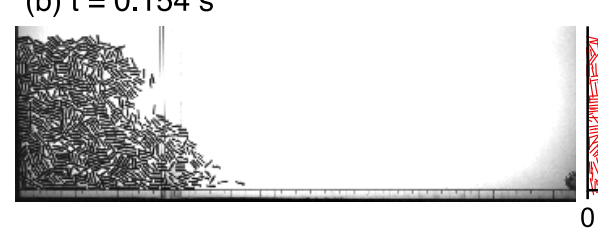

(c) $\mathrm{t}=0.310 \mathrm{~s}$

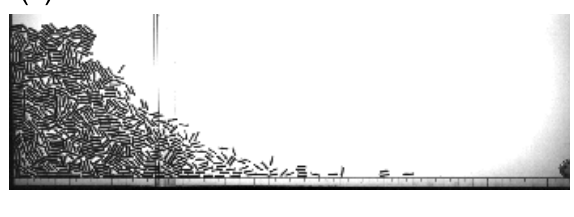

(d) $t=0.468 \mathrm{~s}$

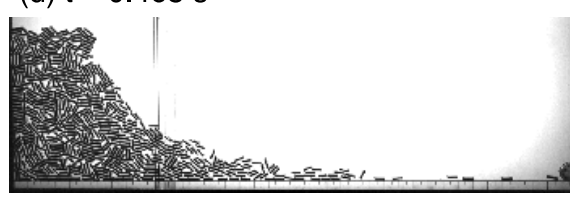

(e) $t=0.622 \mathrm{~s}$

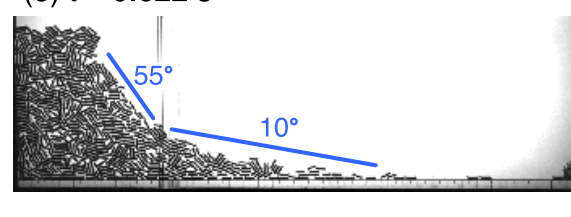

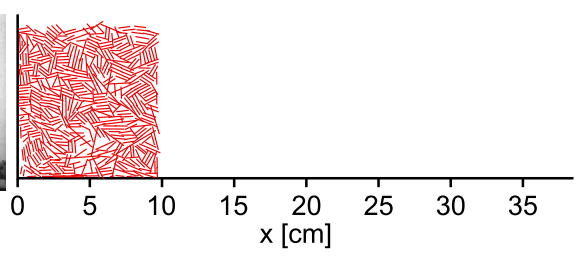
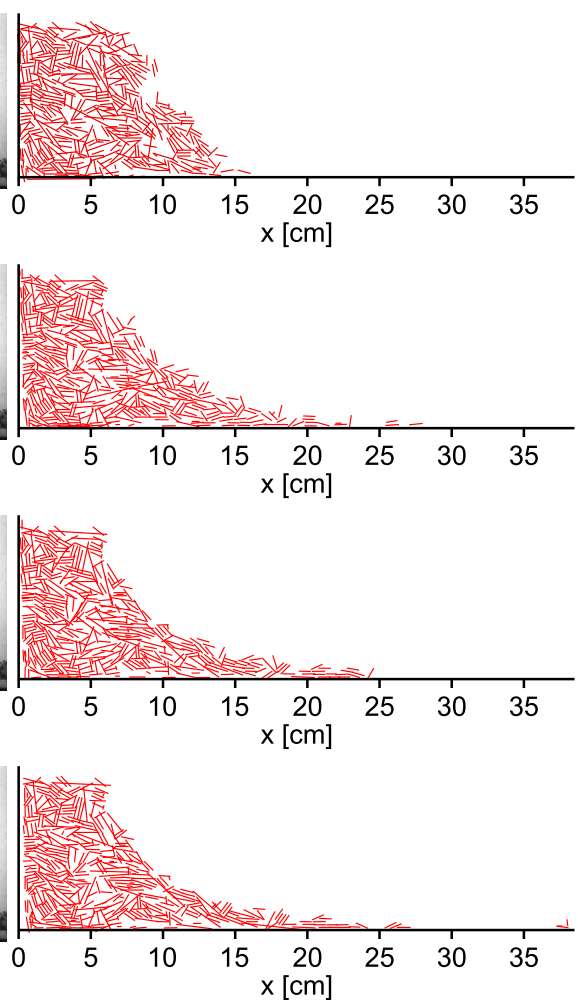

Submerged collapse

(f) $\mathrm{t}=0 \mathrm{~s}$

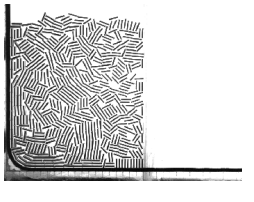

(g) $t=0.390 \mathrm{~s}$
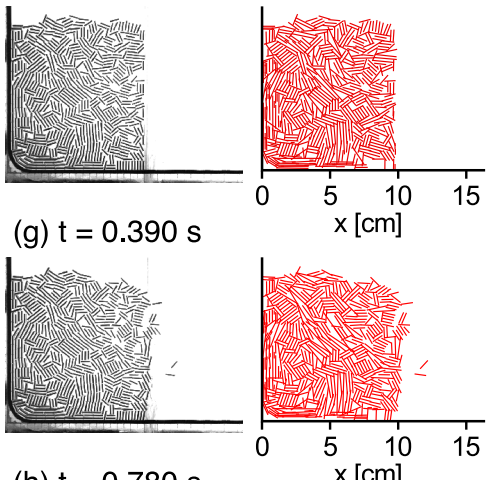

(h) $\mathrm{t}=0.780 \mathrm{~s}$
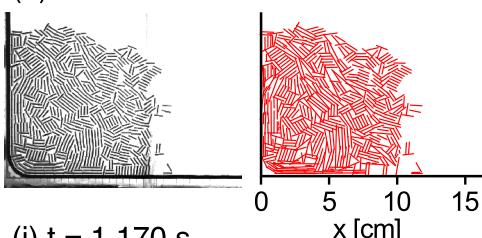

(i) $\mathrm{t}=1.170 \mathrm{~s}$
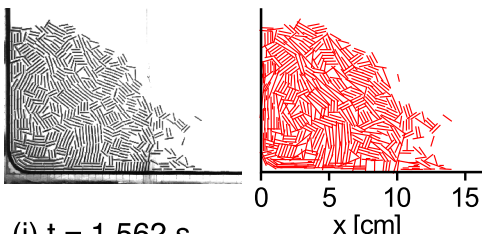

(j) $t=1.562 \mathrm{~s}$

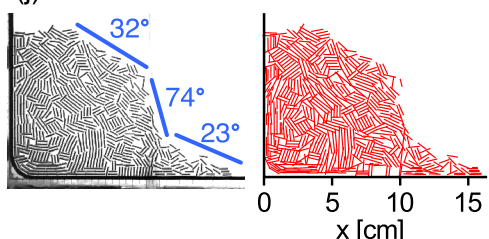

Figure 2. Collapse sequence of a granular column with elongated particles. (a-e) dry column; and (f-j) submerged column. The grey-scale images are photographs from the experiment and the red markers show the grain orientations identified by the digital image analysis. The blue lines indicate the different slopes at the deposit surface $(e, j)$.

cap-to-cap contacts are found, resulting in a single measurement of an artificial longer grain. As a result each image from the initial and final configuration is closely curated, removing inaccurate measurements.

The probability $P_{\vartheta}(\vartheta)$ of grain orientations is computed as

$$
P_{\vartheta}(\vartheta)=\frac{N_{p}(\delta \vartheta)}{N_{p}}
$$

where $N_{p}(\delta \vartheta)$ is the number of grains within the orientation range $\delta \vartheta$, and $N_{p}$ is the total number of grains. The orientational distribution function is obtained as in [12], fitting a harmonic approximation by

$$
P_{\vartheta}(\vartheta)=\frac{1}{2 \pi}\left(1+a_{p} \cos 2\left(\vartheta-\vartheta^{*}\right)\right)
$$

where $a_{p}$ corresponds to the distribution anisotropy and $\vartheta^{*}$ is the distribution peak grain orientation. Figure 3 presents the probability distribution of grain orientation before and after collapse, for all dry and submerged collapses.

On the one hand, the initial configuration of dry columns present an anisotropic distribution, with a dominant orientation close to $\vartheta \approx 0$ and a distribution anisotropy $a_{p}=0.44$. On the other hand, the saturation process and column preparation results in submerged columns with a less anisotropic orientation, with dominant vertical $\vartheta \approx \pi / 2$ and horizontal orientation $\vartheta \approx 0$ and with an isotropic distribution of $a_{p} \approx 0.02$. Given the limited number of grains involved in the released wedge, the global orientation distribution is not completely modified, but a tendency towards more horizontal orientations is noted.

We theorize that columns consisting of a larger amount of grains would develop a deposit with a nearly nematic configuration. This configuration would be achieved easily in submerged conditions, resulting from the higher fluid drag and its orientational action towards a horizontal direction. Future work will explore this relative grain-size effects.

\section{Conclusions and further work}

This paper presents an experimental approach to study the collapse of a column of elongated grains in dry and submerged conditions. The columns are built in a planar 


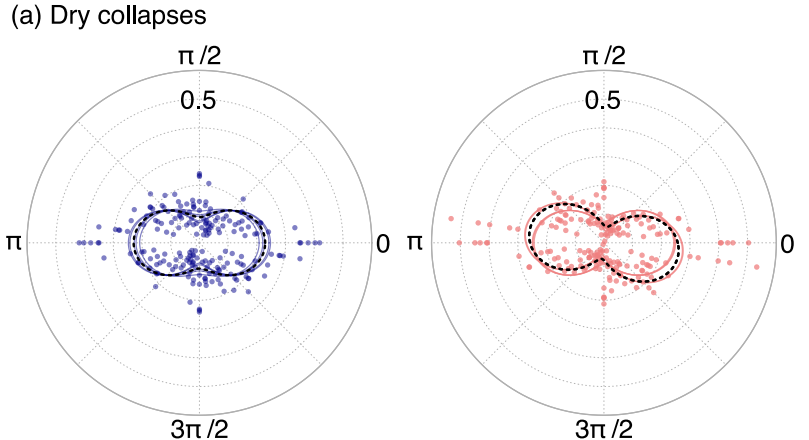

(b) Submerged collapses

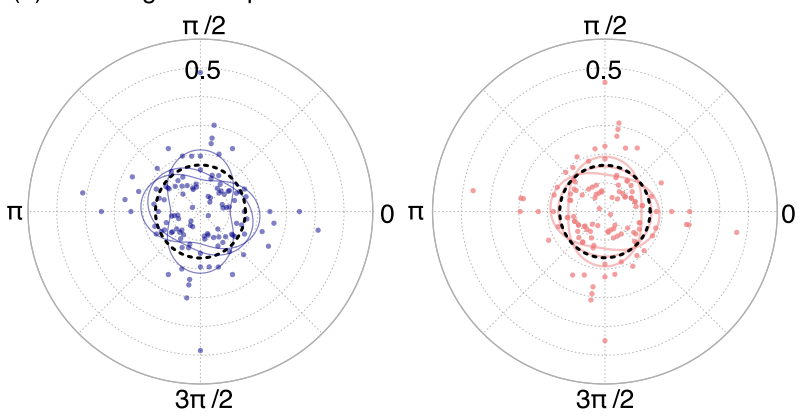

Figure 3. Polar representation of the probability density of grain orientations around an angle $\vartheta$ comparing the initial (right) and final (left) configuration. (a) Orientations for the dry collapses; and (b) Orientations for the submerged collapses. The symbols correspond to $P_{\vartheta}(\vartheta)$, the solid lines present the harmonic fit for each experiment (Eq. 2), and the black dashed line presents the harmonic fit for all tests.

setup, allowing a direct monitoring of the grains motion and orientation during collapse. The column collapse is characterized by the release of a top wedge, falling and spreading laterally, while maintaining an inner and extensive basal stagnant core. The mobilized grains tend to develop an organized orientation, becoming dominant as the result of an increased drag on the submerged free-falling grains. These experimental results are promising in merging information at the grain scale with the fluid patterns and the global column collapse. Overall, the amount of grains within the moving wedge limits the generality of observations, requiring of larger granular systems in order to find conclusive observations regarding the change in grain orientation.

Further efforts need to guarantee comparable initial column configurations in dry and submerged conditions. Moreover, it remains unclear whether a nematic configuration might be achieved in larger systems. Hence, future experiments will test columns with a larger number of grains, extending the experimental observations with numerical simulations. Finally, the setup successfully allows the study of grain shape effects, opening the opportunity for more complex shapes in future works.

\section{Acknowledgments}

We would like to thank Laura Molano for identifying the bugle beads for this series of experiments during her undergrad project.

\section{References}

[1] L. Staron, E.J. Hinch, J. Fluid Mech. 545, 1-27 (2005)

[2] A. Ritter, Zeitschrift des Vereines Deutscher Ingenieure 36, 947 (1892)

[3] A. Schoklitsch, Stitzungsberichte, Mathemat tsch naturwissenschaft- 1 tche Klassee, Akademie der Wissenschaften in Wien 125 (1917)

[4] H.E. Huppert, J.B. Shepherd, R.H. Sigurdsson, S.J. Sparks, J. Volcanol. Geoth. Res. 14, 199 (1982)

[5] G. Lube, H.E. Huppert, R.S.J. Sparks, M.A. Hallworth, J. Fluid Mech. 508, 175-199 (2004)

[6] E. Lajeunesse, A. Mangeney-Castelnau, J.P. Vilotte, Phys. Fluids 16, 2371 (2004)

[7] P.Y. Lagrée, L. Staron, S. Popinet, J. Fluid Mech. 686, 378 (2011)

[8] E.J. Fern, K. Soga, Acta Geotech. 11, 659 (2016)

[9] C. Wang, Y. Wang, C. Peng, X. Meng, Phys. Fluids 29, 103307 (2017)

[10] F. Ceccato, A. Leonardi, V. Girardi, P. Simonini, M. Pirulli, Soils Found. 60, 683 (2020)

[11] M. Cabrera, N. Estrada, Phys. Rev. E 99, 012905 (2019)

[12] E. Azéma, F. Radjaï, Phys. Rev. E 81, 051304 (2010)

[13] M. Trepanier, S.V. Franklin, Phys. Rev. E 82, 011308 (2010)

[14] H. Tapia-McClung, R. Zenit, Phys. Rev. E 85, 061304 (2012)

[15] G. Pinzon, M. Cabrera, Phys. Fluids 31, 086603 (2019)

[16] G. Pinzón, M.A. Cabrera, Submerged planar granular column collapse: Fluid fluxes at the collapsing granular front, in Association of Environmental and Engineering Geologists; special publication 28 (Colorado School of Mines. Arthur Lakes Library, 2018)

[17] M.A. Cabrera, G. Pinzon, W.A. Take, R.P. Mulligan, J. Geophys. Res. Oceans 125, 1 (2020)

[18] G. Pinzon, M. Cabrera, Planar Granular Column Collapse: A Novel Releasing Mechanism, in Proceedings of China-Europe Conferenceon Geotechnical Engineering, edited by W. Wu, H.S. Yu (Springer, 2018), pp. 591-596, https://doi .org/ 10. 1007/978-3-319-97112-4_132

[19] A. Belmonte, H. Eisenberg, E. Moses, Phys. Rev. Lett. 81, 345 (1998)

[20] S. Van der Walt, J.L. Schönberger, J. Nunez-Iglesias, F. Boulogne, J.D. Warner, N. Yager, E. Gouillart, T. Yu, PeerJ 2, e453 (2014)

[21] J. Sauvola, M. Pietikäinen, Pattern Recogn. 33, 225 (2000) 